\title{
Review Article \\ Effect of Different Exercise Modalities on Oxidative Stress: A Systematic Review
}

\author{
Anand Thirupathi $\mathbb{D}^{1}$ Meizi Wang, ${ }^{2}$ Ji Kai Lin, ${ }^{1}$ Gusztáv Fekete, ${ }^{2}$ Bíró István, ${ }^{3}$ \\ Julien S. Baker, ${ }^{4}$ and Yaodong Gu (i) ${ }^{1}$ \\ ${ }^{1}$ Faculty of Sports Science, Ningbo University, Ningbo 315211, China \\ ${ }^{2}$ Savaria Institute of Technology, Eötvös Loránd University, Szombathely 9700, Hungary \\ ${ }^{3}$ Faculty of Engineering, University of Szeged, Szeged, Hungary \\ ${ }^{4}$ Centre for Health and Exercise Science Research, Department of Sport, Physical Education and Health, Hong Kong \\ Baptist University, Hong Kong 999077, China
}

Correspondence should be addressed to Anand Thirupathi; ananthzeal@gmail.com and Yaodong Gu; guyaodong@hotmail.com Received 18 July 2020; Revised 15 December 2020; Accepted 16 January 2021; Published 11 February 2021

Academic Editor: Hugo Olmedillas

Copyright (c) 2021 Anand Thirupathi et al. This is an open access article distributed under the Creative Commons Attribution License, which permits unrestricted use, distribution, and reproduction in any medium, provided the original work is properly cited.

\begin{abstract}
Exercise-induced benefits are being increasingly recognized in promoting health and preventing diseases. However, initial adaption to exercise response can have different effects on cells, including an increase in the formation of oxidants and inflammatory mediators that ultimately leads to oxidative stress, but this scenario depends on the exercise type and intensity and training status of the individual. Therefore, we aimed to understand the effect of different types of exercise on oxidative stress. Indeed, exercise-induced minimum oxidative stress is required for regulating signaling pathways. According to the Preferred Reporting Items for Systematic Reviews and Meta-Analyses (PRISMA) statement, a search for relevant articles was carried out on PubMed/Medline, ISI Web of Science, and Google Scholar using a broad range of synonyms such as oxidants, reactive oxygen species (ROS), oxidative stress, exercise, physical training, aerobic exercise, and strength exercise until 2019. This study selected a total of 18 articles for assessing the oxidative damage using various parameters such as malondialdehyde (MDA), protein carbonyl (PCO), and F1-isoprostanes and enzymatic antioxidants. We observed that any type of exercise can increase the oxidative damage in an exercise type and intensity manner. Further, the training status of the individual and specific oxidative damage marker plays a crucial role in predicting earlier oxidative damage in the exercise condition. However, some of the studies that we included for review did not perform follow-up evaluations. Therefore, follow-up programs using larger numbers need to be performed to confirm our findings.
\end{abstract}

\section{Introduction}

Life expectancy is associated with several factors, and physical exercise is one of the factors that help to promote human life expectancy. Studies have shown that exercise can prevent several life-threatening diseases such as cardiovascular diseases, obesity-related diseases, and some types of cancers [1-3]. Reactive oxygen species (ROS) can be the direct factor or associated factor to cause or prevent these diseases [4]. Research advancements in both exercise and free radical biology have been providing substantial developments in the knowledge related to the mechanisms of exercise and oxi- dative stress [5,6]. Although initial investigations reported the negative effect of ROS, recent studies have shown that exercise-induced ROS can upregulate several enzymatic and nonenzymatic antioxidants in the biological system [7, 8], and exercise could be an optimizer of ROS in negating oxidative damage in the cells, while ROS can regulate signaling or act as a signaling molecule to muscular adaption. In this regard, several studies have concluded that regular physical exercise does not culminate in chronic oxidative stress in the active muscles $[9,10]$. This supports the concept of exercise-induced hormesis. The term hormesis is used to define the biphasic dose-response curve in the biological 
system where a smaller amount of stress can facilitate adaptation whereas chronic and/or high dose of stressor can increase the damage to the cells $[11,12]$. This scenario can play several regulatory roles from genotypic to phenotypic levels. However, this hormesis effect depends on the exercise type and intensity which could be the preserver or spoiler of this condition, and this can demand additional research.

The first study suggests that aerobic exercise can increase oxidative stress [13]. After this, several studies have reported that acute aerobic exercise increases oxidative stress and decreases antioxidant levels [5, 14, 15]. However, certain studies have shown that exercise was observed to influence only some oxidative stress markers or none at all $[16,17]$. Also, studies have shown that anaerobic exercise can increase oxidative stress and decrease or increase antioxidants [18]. Further, whole-body resistance exercise increased oxidative damage. For example, resistance exercise at a 10-repetition maximum load increases the MDA level in the blood [19]. Furthermore, local resistance exercise, which is a single type of resistance training in a specific muscle group, can increase oxidative damage. In contrast, other studies have shown that resistance exercises did not cause oxidative stress, and this may be due to the training status of the individual $[20,21]$. However, changes in the individual variability such as exercise-induced reductive stress or negligible stress have a considerable effect on some people. For example, oxidative stress response is changed in individuals who performed eccentric knee extension even in the same training status [22]. These studies suggest that both aerobic exerciseinduced oxidative stress and anaerobic exercise-induced oxidative stress are attributed not only to the training status of the individual but also to interindividual variability. Therefore, focusing on the exercise type, intensity, and training status and considering wide variability of individuals such as reductive stress may provide better insight into exercise-induced oxidative stress following benefits and/or consequences. The aim of the present study was to establish an understanding of the effect of different types of exercise on oxidative stress.

\section{Methodology}

In accordance with the guidelines for the Preferred Reporting Items for Systematic Reviews and Meta-Analyses (PRISMA) statement, a search for relevant articles was carried out on PubMed/Medline, ISI Web of Science, and Google Scholar terms using a broad range of synonyms including ROS, oxidative damage, oxidants, physical activity, aerobic exercise, and strength exercise until 2019. To avoid the risk of missing relevant articles, additional papers were searched on the grey literature (i.e., generic web search) and through the bibliography of previous reviews. One author (AT) ran the search and screened the initial titles after duplicates were removed. Two authors (AT and GY) independently examined potentially relevant articles in depth. We included only papers published in peer-reviewed journals which reported findings from experimental controlled studies, i.e., human studies only. We excluded articles not available in English, unpublished papers, and conference posters or those reporting findings of nonexperimental studies (e.g., pre- and postintervention studies and case series). The first author's name, year of publication, sample of intervention and control group, design and duration of the study, topic and type of intervention, outcome, assessment, and results were recorded using an electronic spreadsheet.

\section{Results}

3.1. Search Results. Two hundred twenty-eight articles were identified from initial database searches and are presented in Figure 1. After screening was performed using titles and relative keywords, a total of 205 articles were excluded. The remaining 23 potential articles' abstracts were carefully evaluated, and five articles were excluded. The full text of the remaining 18 articles was retrieved and reviewed, which was then included for systemic analysis (Figure 1).

3.2. Participant Characteristics. The number of studies included for review was 18 . The study population, the number of participants, mean age, intervention, and main outcomes are outlined in Table 1.

3.3. Study Selection. This study selected 18 articles for assessing the effect of different exercise protocols on reducing oxidative damage. A total of 17 articles were identified by searching databases, and one was identified by the article's reference for this study. All the records used in this study were on human subjects. Seven studies analyzed the effect of resistance exercise on oxidative damage, and eight studies analyzed the effect of aerobic exercise on oxidative damage. One study analyzed the combined effects of both aerobic and resistance training on oxidative damage. Two studies analyzed the general physical activity outcomes with the aim of reducing oxidative damage.

3.4. Risk of Bias of Included Studies. From the 18 included studies, at least nine studies had a risk of bias. Eight studies had high risk of blinding of participants and outcome assessments (Table 2). One study had a high risk of randomization, and one study had allocation concealment. All the studies included in this study had a low risk of an incomplete outcome. A total of 12 studies had unclear risk in the randomization of participants, and all the 18 included studies had unclear risks associated with other bias (Figure 2).

3.5. Effect of Exercise on Oxidative Stress Markers. We selected a total of eighteen studies for the analysis of exercise effects on reducing oxidative damage. Selected studies used different oxidative damage markers such as malondialdehyde (MDA), protein carbonyl (PC), and isoprostanes and the level of enzymatic antioxidants like superoxide dismutase (SOD), catalase (CAT), total antioxidant capacity (TAC), and glutathione (GSH). Different training sessions were performed by the participants, and most of the studies that we selected belong to the regions of Europe-based countries. Four studies were from Greece, three from Poland, two from Austria, and one from France and Spain. There were three studies from the USA, one from Chile, and one from Brazil. There were also two studies from Middle East countries, Iran, 


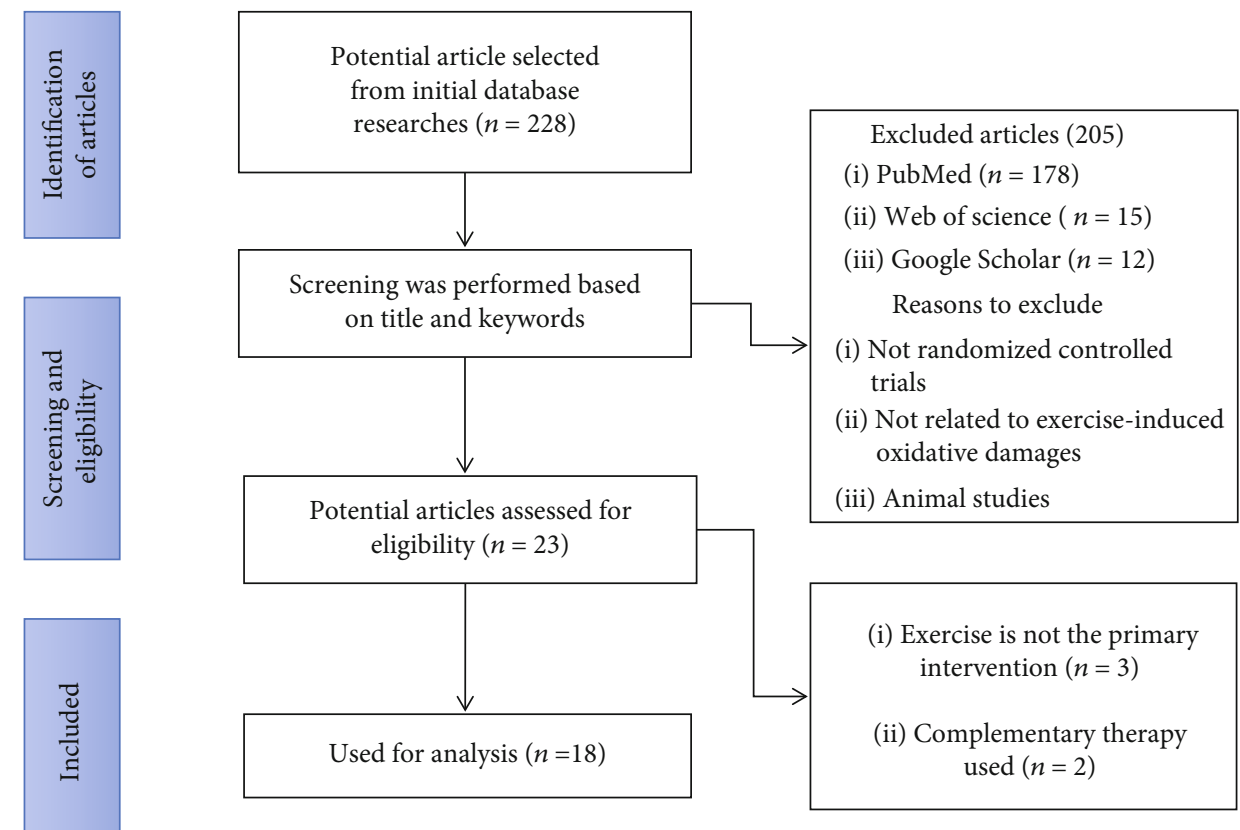

FIgURE 1: The search flowchart for the screening process.

and Turkey. One study used incremental exercise to perform moderate concentric and high-intensity eccentric exercise to analyze the oxidative damage [23]. The study found that moderate-intensity exercise did not increase oxidative stress. However, high-intensity exercise increased oxidative stress and muscle damage. Well-trained individuals were able to cope with the oxidative stress easily during eccentric exercise [41]. A further study found that no resistance training increased oxidative stress with the participants [24]. In contrast, other studies found that different combinations of resistance training combat the oxidative damages than usual resistance training $[25,34]$, whereas another study observed that circuit training decreased the oxidative damage after exercise [36]. Also, a study found that endurance training decreased oxidative stress and increased antioxidant status [27]. One session of high-intensity training (HIT) increased the oxidative stress along with antioxidants whereas a short session decreased the oxidative stress-induced damage along with improving antioxidants [29]. A further study also found that repeated bouts of exercises increased the oxidative stress-induced damage more than the first bout [31]. Different training modalities like running, ski running, and football playing increase the ROS generation along with antioxidants [32] whereas other studies found that increased maximal $\mathrm{O}_{2}$ uptake decreased the F2-isoprostane, a marker of oxidative damage $[33,35]$. Other studies found that even low levels of physical activity such as fishing, long walking, team games, and picking mushrooms can reduce the oxidative stressinduced damage, but oxidative stress-induced damage was intensified with aging [38]. Another study found that both aerobic and resistance exercises increased the oxidative stressinduced damage [39], while one study found that resistance training minimally affected DNA oxidation and lipid profiles [28]. In one study, we analyzed only resistance training effects on oxidative stress which had other exercise components including cognitive ability upregulation [34]. In another study, we included only active young individuals in the analysis [30]. Most of the studies did not use any control groups; instead, they used pre- and postexercise values for analyzing the effect of exercise on oxidative damage [23, 24, 27-29, 31, 32, 36-38].

\section{Discussion}

Exercise-induced oxidative stress is an important factor in deciding both benefits and consequences including regulating signaling pathways or acting as a signaling molecule, biogenesis, and causing damages to the cells [11]. However, the way how exercise performance could affect the abovementioned condition is ambiguous. Therefore, additional attention is necessary while prescribing exercise to the individual. This study systematically analyzed the different exercise training effects on oxidative stress condition, and we observed that any type of exercise can increase or decrease oxidative damage according to the exercise type, intenstiy and training status, and specific oxidative damage markers that are used to measure the oxidative damage. This study analyzed a total of 18 articles that focused on effect of exercise on reducing oxidative damage. Eight articles were selected for the effect of resistance training on oxidative stress biomarkers such as MDA and 8-hydroxydeoxyguanosine along with different antioxidant levels. Studies have shown that resistance training promotes muscle performance by reducing oxidative stress-induced consequences [6]. However, this study found that resistance training had no effect on reducing oxidative stress, whereas different combinations of resistance exercise had good effect on combating oxidative stress-related damages, and this could be due to specific oxidative damage markers and training status of the individual. Lima et al. [41] have shown that a single session of combined exercise reduced oxidative stress. Gomes et al. [42] have also shown 


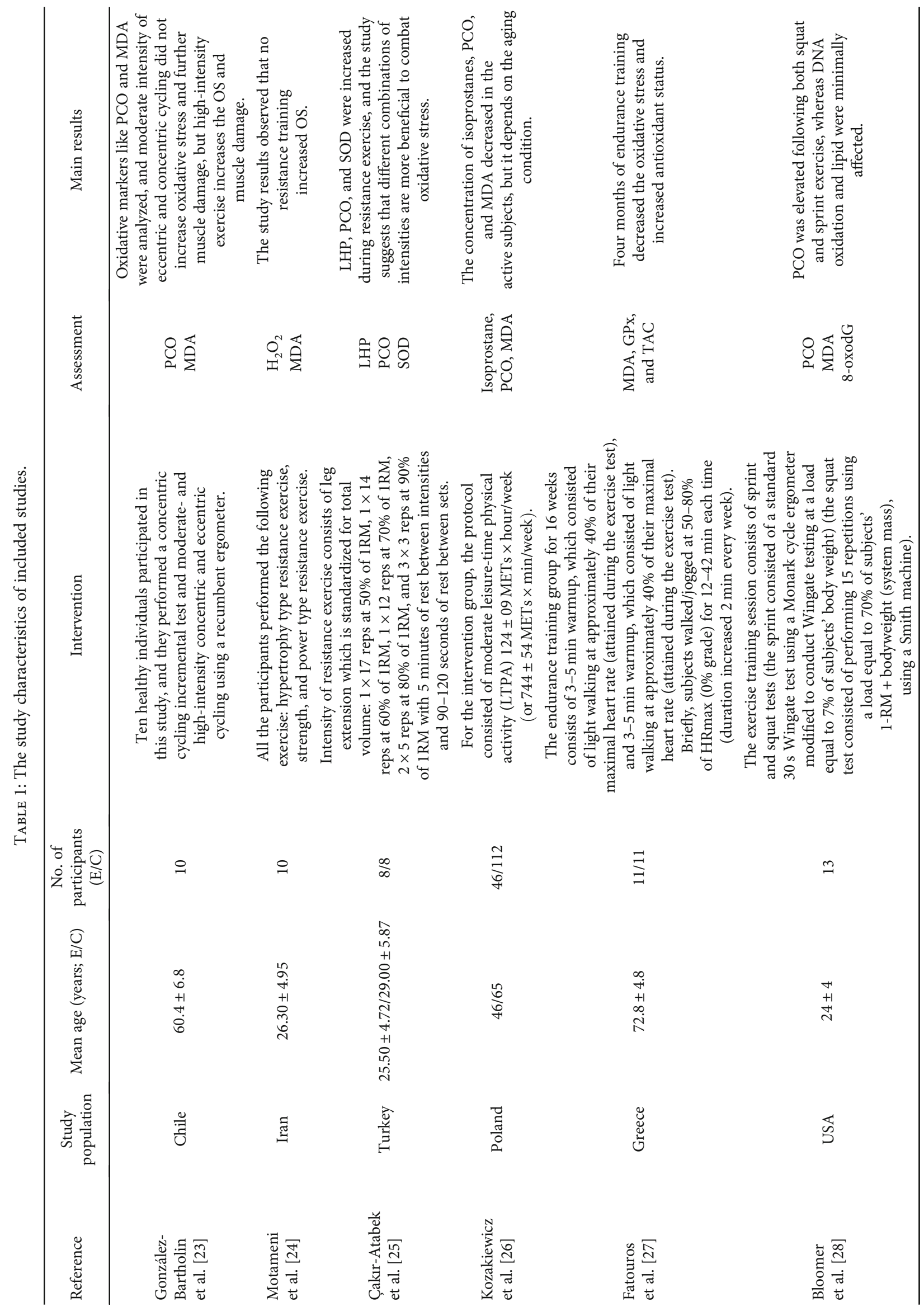




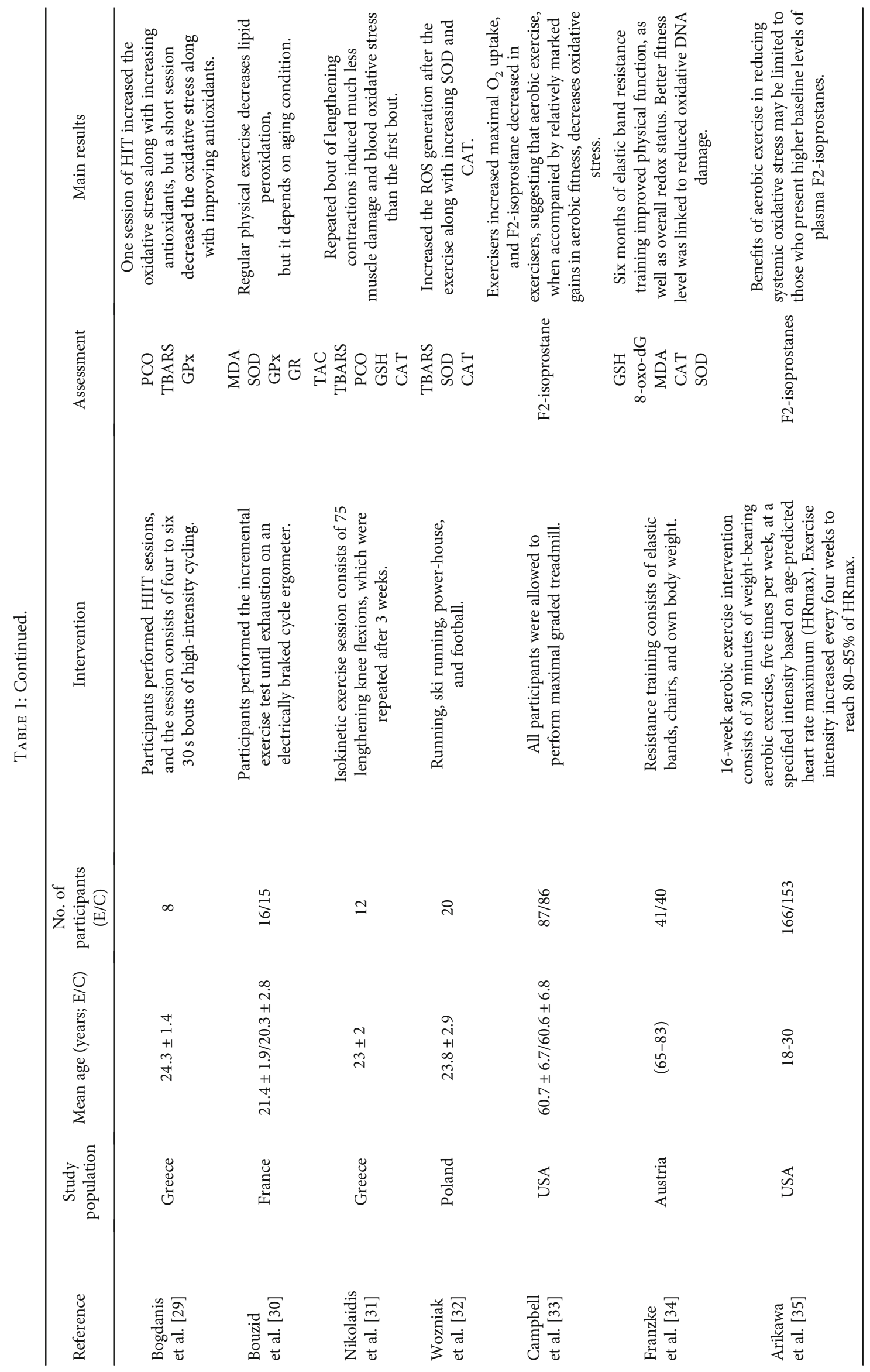




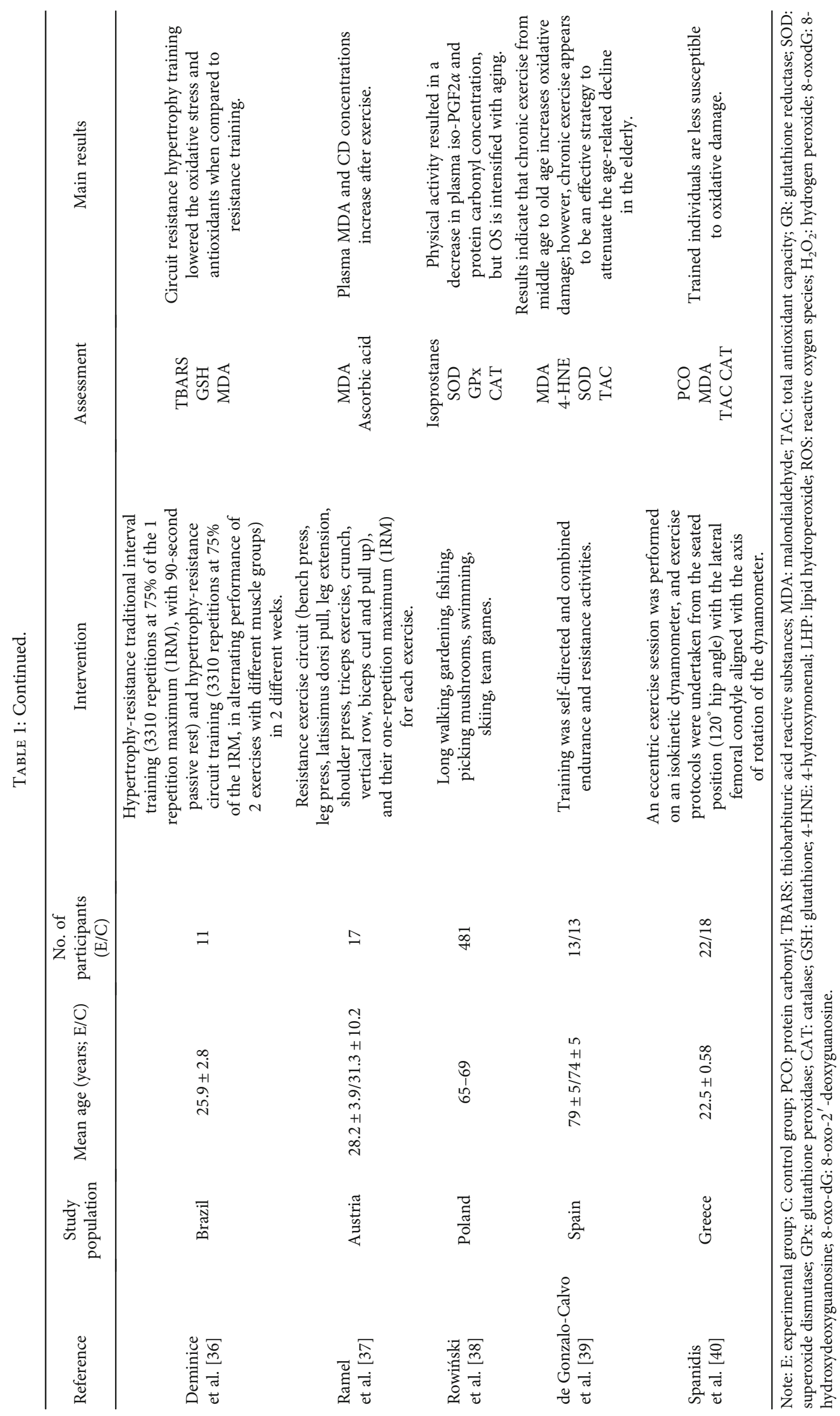


TABLE 2: Risk of bias evaluation of included studies.

\begin{tabular}{|c|c|c|c|c|c|c|c|}
\hline Reference & $\begin{array}{c}\text { Random } \\
\text { sequence } \\
\text { generation }\end{array}$ & $\begin{array}{c}\text { Allocation } \\
\text { concealment }\end{array}$ & $\begin{array}{c}\text { Blinding of } \\
\text { participants and } \\
\text { personnel }\end{array}$ & $\begin{array}{l}\text { Blinding of } \\
\text { outcome } \\
\text { assessment }\end{array}$ & $\begin{array}{c}\text { Incomplete } \\
\text { outcome data }\end{array}$ & $\begin{array}{l}\text { Selective } \\
\text { reporting }\end{array}$ & $\begin{array}{l}\text { Other } \\
\text { bias }\end{array}$ \\
\hline Calabrese and Baldwin [12] & Unclear & Low & High & Low & Low & Low & Unclear \\
\hline Dillard et al. [13] & Unclear & Low & Low & Low & Low & Low & Unclear \\
\hline Lovlin et al. [14] & Unclear & Unclear & High & High & Low & Low & Unclear \\
\hline Powers et al. [5] & Unclear & Unclear & Unclear & Unclear & Unclear & Unclear & Unclear \\
\hline Quindry et al. [15] & Low & Low & Low & Low & Low & Low & Unclear \\
\hline Alessio et al. [16] & Unclear & Unclear & High & High & Low & Low & Unclear \\
\hline Bloomer et al. [17] & Unclear & Low & High & High & Low & Low & Unclear \\
\hline Marzatico et al. [18] & Unclear & Low & High & High & Low & Low & Unclear \\
\hline McBride et al. [19] & Unclear & Low & Low & Low & Low & Low & Unclear \\
\hline Hoffman et al. [20] & Unclear & Unclear & Unclear & Unclear & Low & Low & Unclear \\
\hline Bloomer et al. [21] & Low & Low & Low & Low & Low & Low & Unclear \\
\hline Margaritelis et al. [22] & Low & Low & Low & Low & Low & Low & Unclear \\
\hline González-Bartholin et al. [23] & Unclear & Low & High & High & Low & Low & Unclear \\
\hline Motameni et al. [24] & Low & Unclear & High & Unclear & Low & Low & Unclear \\
\hline Çakır-Atabek et al. [25] & High & High & Low & Low & Unclear & Low & Unclear \\
\hline Kozakiewicz et al. [26] & Unclear & Unclear & Unclear & Low & Low & Low & Unclear \\
\hline Fatouros et al. [27] & Low & Low & Unclear & High & Unclear & Low & Unclear \\
\hline Bloomer et al. [28] & Unclear & Unclear & Unclear & Unclear & Unclear & Unclear & Unclear \\
\hline
\end{tabular}

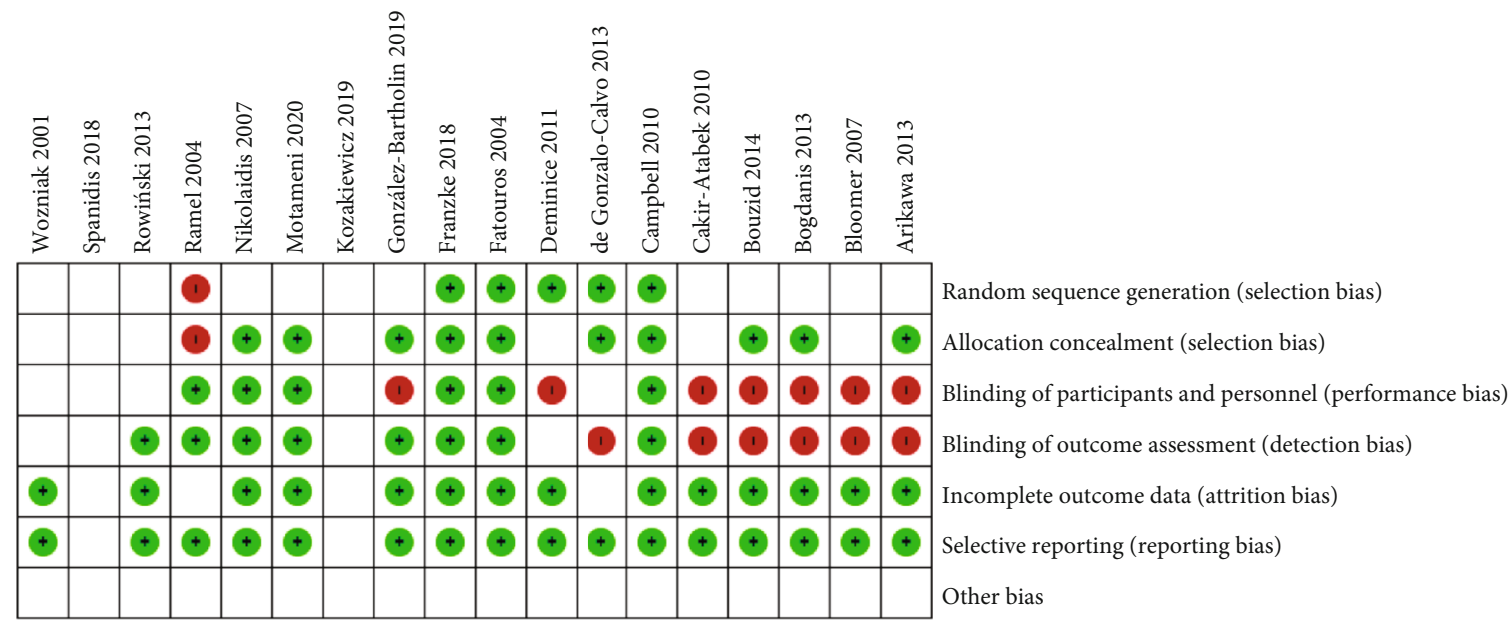

FIGURE 2: Risk of bias evaluation of the included studies.

that aerobic exercise is more superior in combating oxidative stress than resistance-type exercise. Further, some studies observed that well-trained exercise condition can even overcome high-intensity-induced oxidative damage in both aerobic and resistance exercise $[43,44]$.

4.1. Exercise Intensity and Oxidative Stress. Although increased intensity of exercise shifts the redox balance in favor of oxidative stress, this situation is necessary for initial adaption, supporting the concept of exercise-induced hormesis. Higher intensity exercise increases the total antioxidant capacity when compared to low and moderate intensities of treadmill exercise with no changes in lipid peroxidation
[45]. Previously, it was believed that exercise with increased intensity must be overwhelmed by antioxidant defenses which induce a condition of oxidative stress [46]. However, recent reports suggested that even low or moderate intensity can induce oxidative stress, suggesting that exercise volume (duration $\times$ intensities) and failed antioxidant defense system are the primary mediators of exercise-induced oxidative stress. We found that different intensities of resistance training can effectively reduce the oxidative damage in the participants when compared to the same intensities. This could be due to exercise-induced redox-linked health adaptations through upregulating the antioxidant defense system [47]. Regarding aerobic exercise, moderate intensity improved the 
exercise performance by reducing oxidative damage [23]. However, short-term aerobic exercise with higher intensities can greatly induce oxidative damage, but this effect is not extended up to the DNA level [29]. The exercise type is an important factor for inducing oxidative damage because high intensity of cycling reduces the oxidative damage and increases the enzymatic antioxidants [29], but the same intensity with sprint exercise increases oxidative damage [28]. Similarly, resistance trainings such as circuit resistance training reduced the oxidative damage and improved the antioxidant level when compared to traditional resistance training, suggesting that the exercise type along with a total volume of exercise is important for exercise-induced oxidative stress. Other studies have shown that exercise volume is not enough to exhaust antioxidants and increase oxidative stress [48]. The disparity between these studies is due to exercise protocol duration, exercise mode, and the oxidative damage markers analyzed [49-51]. In the current study, different intensities particularly higher intensities with different modes of exercise can greatly reduce oxidative stress by stimulating the plasma antioxidants and subsequent ROS scavenging activities.

4.2. Effect of Different Exercises in Aging. Aging is associated with increased formation of ROS subsequently causing oxidative modification of protein, lipid, and DNA. The reason may be due to defects in the mitochondrial electron transport chain (ETC) and changes in the enzymatic activities of ETC. Consequently, this increases the leakage of electrons and formation of superoxide anion [52]. Physical exercise has several health benefits, but higher intensity induces muscle oxygen flux which ultimately increases oxidative injury. Further, biochemical changes due to aging can facilitate the increase in the formation of ROS and decrease in the muscle repair and regeneration capacity in the aging community. Therefore, it is important to consider several aspects before advising exercise for aged people. Chronic exercise is an effective strategy for reducing age-induced oxidative stress. Even any kind of physical activity is linked with a reduced level of oxidative stress in the elderly such as gaming, fishing, and gardening. This study found that normal physical activity such as fishing or gardening and both combined training of aerobic and resistance training can increase the antioxidants and reduce the oxidative damage in the elderly. However, exercise-reduced oxidative damage in aging may depend on the type of exercise and intensities [30, 38, 39]. For example, single bout of exercise can abruptively exceed a certain intensity or duration resulting in increased production of ROS whereas regular exercise improves the antioxidant defense system with tolerable oxidative damage which in turn induces significant adaptions. However, this can be related to exercise intensity. Bouzid et al. [30] have shown that moderate to higher intensity regular exercise can help to maintain better antioxidants in the elderly. This may be due to different oxidative stress training adaptation that is necessary for older tissues to prevent age-related diseases and to retard the aging process [53]. This preventative effect of aging is achieved through the formation of ROS in part or full to alter the signaling pathways and/or cause molecular damage that can produce adaptive responses to withstand further stress [54]. This study also corroborates with the above statements that different exercise or physical activity can prevent oxidative damage and upregulate antioxidants.

4.3. Methodological Limitation. In addition to fixing the exercise type and intensity to prevent oxidative damage, measuring ROS in the biological system is more complicated since they are very short-lived and highly reactive. Assessing exercise-induced oxidative damage used different oxidative damage markers, and these oxidative damage markers can only be reflected on specific local oxidative stress, but exercise-induced physiological response can occur in the entire system. This questioned the suitability of those markers in assessing the oxidative damage in the exercise condition. Next, sample stability during exercise is an important factor to consider because there is a possibility of the sample to become more viable to oxidative damage. In this aspect, the urinary sample is more convenient even for collection and provide better redox balance for a longer period when compared to blood samples in the exercise condition. However, only some oxidative damage markers are standardized in animals and humans, and this has to be done in the exercised samples with different training status.

4.4. Future Directions. Although several studies have reported that any type of exercise can greatly increase oxidative stress in a type- and intensity-dependent manner, other aspects such as specific oxidative damage markers, reductive stress, and training status of the individual should also need additional research. For example, some promising markers are underway such as acrolein-lysine which will diversify the current parameters to measure the oxidative damage. Further, the status of antioxidant defense during or after exercise is varied which could conflict the measurement of oxidative stress. Therefore, some integrative approaches such as OXY-SCORE or oxidative-INDEX computed by subtracting antioxidative capacity from ROS levels/ROS-induced damage or oxidative stress index (OSI) are required for the measurement of oxidative stress. Taken together, the measurement of oxidative stress during different types of exercise needs reliable research work that should predict earlier oxidative damage in the exercise condition.

\section{Conclusion}

This systematic review observed that any type of exercise or physical activity can greatly increase or decrease oxidative damage in an exercise type and intensity manner and specific oxidative damage markers that are used to measure the oxidative damage. This could cause various misjudgment in predicting oxidative damage in the exercised condition. Further, the data analyzed in this study did not provide conclusive evidence related to the methodology to reduce and adapt to oxidative stress. This was partly due to the fact that studies reviewed did not complete follow-up evaluations. Therefore, different types of exercise using different intensities and durations need to be completed with follow-up programs, with large numbers to make full conclusions about reducing oxidative stress damage. 


\section{Conflicts of Interest}

The authors declare that there is no conflict of interest.

\section{Acknowledgments}

The authors would like to acknowledge Mr. Kishore Kumar who helped in editing the manuscript.

\section{References}

[1] S. N. Blair, Y. Cheng, and J. S. Holder, "Is physical activity or physical fitness more important in defining health benefits?," Medicine and Science in Sports and Exercise, vol. 33, pp. S379-S399, 2001.

[2] C. J. Crespo, M. R. Palmieri, R. P. Perdomo et al., "The relationship of physical activity and body weight with all-cause mortality: results from the Puerto Rico Heart Health Program," Annals of Epidemiology, vol. 12, no. 8, pp. 543-552, 2002.

[3] T. Senoner and W. Dichtl, "Oxidative stress in cardiovascular diseases: still a therapeutic target?," Nutrients, vol. 11, no. 9, p. 2090, 2019.

[4] W. Dröge, "Free radicals in the physiological control of cell function," Physiological Reviews, vol. 82, no. 1, pp. 47-95, 2002.

[5] S. K. Powers, Z. Radak, and L. L. Ji, "Exercise-induced oxidative stress: past, present and future," Journal of Physiology, vol. 594, no. 18, pp. 5081-5092, 2016.

[6] A. Thirupathi and R. A. Pinho, "Effects of reactive oxygen species and interplay of antioxidants during physical exercise in skeletal muscles," Journal of Physiology and Biochemistry, vol. 74, no. 3, pp. 359-367, 2018.

[7] L. L. Ji, "Antioxidants and oxidative stress in exercise," Proceedings of the Society for Experimental Biology and Medicine, vol. 222, no. 3, pp. 283-292, 2003.

[8] K. Fisher-Wellman, H. K. Bell, and R. J. Bloomer, "Oxidative stress and antioxidant defense mechanisms linked to exercise during cardiopulmonary and metabolic disorders," Oxidative Medicine and Cellular Longevity, vol. 2, Article ID 193520, 9 pages, 2009.

[9] S. Di Meo, G. Napolitano, and P. Venditti, "Mediators of physical activity protection against ROS-linked skeletal muscle damage," International Journal of Molecular Sciences, vol. 20, no. 12, p. 3024, 2019.

[10] Z. Radak, K. Ishihara, E. Tekus et al., "Exercise, oxidants, and antioxidants change the shape of the bell-shaped hormesis curve," Redox Biology, vol. 12, pp. 285-290, 2017.

[11] S. K. Powers, R. Deminice, M. Ozdemir, T. Yoshihara, M. P. Bomkamp, and H. Hyatt, "Exercise-induced oxidative stress: friend or foe?," Journal of Sport and Health Science, vol. 9, no. 5, pp. 415-425, 2020.

[12] E. J. Calabrese and L. A. Baldwin, "Chemical hormesis: its historical foundations as a biological hypothesis," Toxicologic Pathology, vol. 27, no. 2, pp. 195-216, 2016.

[13] C. J. Dillard, R. E. Litov, W. M. Savin, E. E. Dumelin, and A. L. Tappel, "Effects of exercise, vitamin E, and ozone on pulmonary function and lipid peroxidation," Journal of Applied Physiology Respiratory Environmental and Exercise Physiology, vol. 45, no. 6, pp. 927-932, 1978.
[14] R. Lovlin, W. Cottle, I. Pyke, M. Kavanagh, and A. N. Belcastro, "Are indices of free radical damage related to exercise intensity," European Journal of Applied Physiology and Occupational Physiology, vol. 56, no. 3, pp. 313-316, 1987.

[15] J. Quindry, C. Dumke, D. Slivka, and B. Ruby, "Impact of extreme exercise at high altitude on oxidative stress in humans," Journal of Physiology, vol. 594, no. 18, pp. 50935104, 2016.

[16] H. M. Alessio, A. H. Goldfarb, and G. Cao, "Exercise-induced oxidative stress before and after vitamin C supplementation," International Journal Sport Nutrition, vol. 7, no. 1, pp. 1-9, 1997.

[17] R. J. Bloomer, M. J. Falvo, A. C. Fry, B. K. Schilling, W. A. Smith, and C. A. Moore, "Oxidative stress response in trained men following repeated squats or sprints," Medicine \& Science in Sports \& Exercise, vol. 38, no. 8, pp. 1436-1442, 2006.

[18] F. Marzatico, O. Pansarasa, L. Bertorelli, L. Somenzini, and G. Della Valle, "Blood free radical antioxidant enzymes and lipid peroxides following long-distance and lactacidemic performances in highly trained aerobic and sprint athletes," Journal of Sports Medicine and Physical Fitness, vol. 37, no. 4, pp. 235-239, 1997.

[19] J. M. McBride, W. J. Kraemer, T. Triplett-McBride, and W. Sebastianelli, "Effect of resistance exercise on free radical production," Medicine \& Science in Sports \& Exercise, vol. 30, no. 1, pp. 67-72, 1998.

[20] J. R. Hoffman, J. Im, J. Kang et al., "Comparison of low- and high-intensity resistance exercise on lipid peroxidation," Journal of Strength and Conditioning Research, vol. 21, no. 1, pp. 118-122, 2007.

[21] R. J. Bloomer, A. H. Goldfarb, and M. J. McKenzie, "Oxidative stress response to aerobic Exercise," Medicine \& Science in Sports \& Exercise, vol. 38, no. 6, pp. 1098-1105, 2006.

[22] N. V. Margaritelis, A. Kyparos, V. Paschalis et al., "Reductive stress after exercise: the issue of redox individuality," Redox Biology, vol. 19, no. 2, pp. 520-528, 2014.

[23] R. González-Bartholin, K. Mackay, D. Valladares, H. ZbindenFoncea, K. Nosaka, and L. Peñailillo, "Changes in oxidative stress, inflammation and muscle damage markers following eccentric versus concentric cycling in older adults," European Journal of Applied Physiology, vol. 119, no. 10, pp. 23012312, 2019.

[24] S. Motameni, H. TaheriChadorneshin, and A. Golestani, "Comparing the effects of resistance exercise type on serum levels of oxidative stress and muscle damage markers in resistance-trained women," Sport Science for Health, vol. 16, no. 3, pp. 443-450, 2020.

[25] H. Çakır-Atabek, F. Özdemir, and R. Çolak, "Oxidative stress and antioxidants responses to progressive resistance exercise intensity in trained and untrained males," Biology of Sport, vol. 32, no. 4, pp. 321-328, 2015.

[26] M. Kozakiewicz, R. Rowiński, M. Kornatowski, A. Dąbrowski, K. Kędziora-Kornatowska, and A. Strachecka, "Relation of moderate physical activity to blood markers of oxidative stress and antioxidant defense in the elderly," Oxidative Medicine and Cellular Longevity, vol. 2019, Article ID 5123628, 7 pages, 2019.

[27] I. G. Fatouros, A. Z. Jamurtas, V. Villiotou et al., "Oxidative stress responses in older men during endurance training and detraining," Medicine and Science in Sports and Exercise, vol. 36, no. 12, pp. 2065-2072, 2004. 
[28] R. J. Bloomer, A. C. Fry, M. J. Falvo, and C. A. Moore, "Protein carbonyls are acutely elevated following single set anaerobic exercise in resistance trained men," Journal of Science and Medicine in Sport, vol. 10, no. 6, pp. 411-417, 2007.

[29] G. C. Bogdanis, P. Stavrinou, I. G. Fatouros et al., "Short-term high-intensity interval exercise training attenuates oxidative stress responses and improves antioxidant status in healthy humans," Food and Chemical Toxicology, vol. 61, pp. 171177, 2013.

[30] M. A. Bouzid, O. Hammouda, R. Matran, S. Robin, and C. Fabre, "Changes in oxidative stress markers and biological markers of muscle injury with aging at rest and in response to an exhaustive exercise," PLoS One, vol. 9, no. 3, article e90420, 2014.

[31] M. G. Nikolaidis, V. Paschalis, G. Giakas et al., "Decreased blood oxidative stress after repeated muscle-damaging exercise," Medicine and Science in Sports and Exercise, vol. 39, no. 7, pp. 1080-1089, 2007.

[32] A. Wozniak, G. Drewa, G. Chesy, A. Rakowski, M. Rozwodowska, and D. Olszewska, "Effect of altitude training on the peroxidation and antioxidant enzymes in sportsmen," Medicine and Science in Sports and Exercise, vol. 33, no. 7, pp. 1109-1113, 2001.

[33] P. T. Campbell, M. D. Gross, J. D. Potter et al., "Effect of exercise on oxidative stress," Medicine and Science in Sports and Exercise, vol. 42, no. 8, pp. 1448-1453, 2010.

[34] B. Franzke, B. Schober-Halper, M. Hofmann et al., "Age and the effect of exercise, nutrition and cognitive training on oxidative stress - the Vienna Active Aging Study (VAAS), a randomized controlled trial," Free Radical Biology and Medicine, vol. 121, pp. 69-77, 2018.

[35] A. Y. Arikawa, W. Thomas, M. Gross et al., "Aerobic training reduces systemic oxidative stress in young women with elevated levels of $\mathrm{F}_{2}$-isoprostanes," Contemporary Clinical Trials, vol. 34, no. 2, pp. 212-217, 2013.

[36] R. Deminice, T. Sicchieri, M. S. Mialich, F. Milani, P. P. Ovidio, and A. A. Jordao, "Oxidative stress biomarker responses to an acute session of hypertrophy-resistance traditional interval training and circuit training," Journal of Strength and Conditioning Research, vol. 25, no. 3, pp. 798-804, 2011.

[37] A. Ramel, K. H. Wagner, and I. Elmadfa, "Plasma antioxidants and lipid oxidation after submaximal resistance exercise in men," European Journal of Nutrition, vol. 43, no. 1, pp. 2-6, 2004.

[38] R. Rowiński, M. Kozakiewicz, K. Kędziora-Kornatowska, E. Hübner-Woźniak, and J. Kędziora, "Markers of oxidative stress and erythrocyte antioxidant enzyme activity in older men and women with differing physical activity," Experimental Gerontology, vol. 48, no. 11, pp. 1141-1146, 2013.

[39] D. de Gonzalo-Calvo, B. Fernández-García, B. de Luxán-Delgado et al., "Chronic training increases blood oxidative damage but promotes health in elderly men," Age (Dordrecht, Netherlands), vol. 35, no. 2, pp. 407-417, 2013.

[40] Y. Spanidis, D. Stagos, C. Papanikolaou et al., "Resistancetrained individuals are less susceptible to oxidative damage after eccentric exercise," Oxidative Medicen and Cellular Longevity, vol. 2018, article 6857190, 11 pages, 2018.

[41] A. H. R. A. Lima, M. A. Correia, A. H. G. Soares et al., "Acute effects of walking and combined exercise on oxidative stress and vascular function in peripheral artery disease," Clinical Physiology and Functional Imaging, vol. 38, no. 1, pp. 69-75, 2018.
[42] M. J. Gomes, L. U. Pagan, A. R. R. Lima et al., "Effects of aerobic and resistance exercise on cardiac remodelling and skeletal muscle oxidative stress of infarcted rats," Journal of Cellular and Molecular Medicine, vol. 24, no. 9, pp. 5352-5362, 2020.

[43] S. Y. Park and Y. S. Kwak, "Impact of aerobic and anaerobic exercise training on oxidative stress and antioxidant defense in athletes," Journal of exercise rehabilitation, vol. 12, no. 2, pp. 113-118, 2016.

[44] L. Paes, D. Lima, C. Matsuura et al., "Effects of moderate and high intensity isocaloric aerobic training upon microvascular reactivity and myocardial oxidative stress in rats," PLoS One, vol. 15, no. 2, article e0218228, 2020.

[45] C. D. Schneider, J. Barp, J. L. Ribeiro, A. Bello-Klein, and A. R. Oliveira, "Oxidative stress after three different intensities of running," Canadian Journal of Applied Physiology, vol. 30, no. 6, pp. 723-734, 2005.

[46] J. Vina, M. C. Gomez-Cabrera, A. Lloret et al., "Free radicals in exhaustive physical exercise: mechanism of production, and protection by antioxidants," IUBMB Life, vol. 50, no. 4, pp. 271-277, 2000.

[47] Z. Radak, Z. Zhao, E. Koltai, H. Ohno, and M. Atalay, “Oxygen consumption and usage during physical exercise: the balance between oxidative stress and ROS-dependent adaptive signaling," Antioxidants \& Redox Signaling, vol. 18, no. 10, pp. 1208-1246, 2013.

[48] L. Parker, T. A. McGuckin, and A. S. Leicht, "Influence of exercise intensity on systemic oxidative stress and antioxidant capacity," Clinical Physiology and Functional Imaging, vol. 34, no. 5, pp. 377-383, 2014.

[49] C. Goto, Y. Higashi, M. Kimura et al., "Effect of different intensities of exercise on endothelium-dependent vasodilation in humans," Circulation, vol. 108, no. 5, pp. 530-535, 2003.

[50] C. Goto, K. Nishioka, T. Umemura et al., "Acute moderateintensity exercise induces vasodilation through an increase in nitric oxide bioavailiability in humans," American Journal of Hypertension, vol. 20, no. 8, pp. 825-830, 2007.

[51] D. A. Leaf, M. T. Kleinman, M. Hamilton, and T. J. Barstow, "The effect of exercise intensity on lipid peroxidation," Medicine \& Science in Sports \& Exercise, vol. 29, no. 8, pp. 10361039, 1997.

[52] L. L. Ji, "Exercise at old age: does it increase or alleviate oxidative stress?," Annals of the New York Academy of Sciences, vol. 928, pp. 236-247, 2001.

[53] Z. Radak, H. Y. Chung, and S. Goto, "Exercise and hormesis: oxidative stress-related adaptation for successful aging," Biogerontology, vol. 6, no. 1, pp. 71-75, 2005.

[54] S. Bayod, C. Guzmán-Brambila, S. Sanchez-Roige et al., "Voluntary exercise promotes beneficial anti-aging mechanisms in SAMP8 female brain," Journal of Molecular Neuroscience, vol. 55, no. 2, pp. 525-532, 2015. 